\title{
Adjustments to Model Predictions of Depth of Shower Maximum and Signals at Ground Level using Hybrid Events of the Pierre Auger Observatory
}

\author{
Jakub Vícha ${ }^{a, *}$ on behalf of the Pierre Auger ${ }^{b}$ Collaboration \\ (a complete list of authors can be found at the end of the proceedings) \\ ${ }^{a}$ Institute of Physics of the Czech Academy of Sciences, Prague, Czech Republic \\ ${ }^{b}$ Observatorio Pierre Auger, Av. San Martín Norte 304, 5613 Malargüe, Argentina \\ E-mail: spokespersons@auger.org
}

\begin{abstract}
We present a new method to explore simple ad-hoc adjustments to the predictions of hadronic interaction models to improve their consistency with observed two-dimensional distributions of the depth of shower maximum, $X_{\max }$, and signal at ground level, as a function of zenith angle. The method relies on the assumption that the mass composition is the same at all zenith angles, while the atmospheric shower development and attenuation depend on composition in a correlated way. In the present work, for each of the three leading LHC-tuned hadronic interaction models, we allow a global shift $\Delta X_{\max }$ of the predicted shower maximum, which is the same for every mass and energy, and a rescaling $R_{\text {Had }}$ of the hadronic component at ground level which depends on the zenith angle.

We apply the analysis to 2297 events reconstructed by both fluorescence and surface detectors at the Pierre Auger Observatory with energies $10^{18.5}-10^{19.0} \mathrm{eV}$. Given the modeling assumptions made in this analysis, the best fit reaches its optimum value when shifting the $X_{\max }$ predictions of hadronic interaction models to deeper values and increasing the hadronic signal at both extreme zenith angles. The resulting change in the composition towards heavier primaries alleviates the previously identified model deficit in the hadronic signal (commonly called the muon deficit), but does not remove it. Because of the size of the required corrections $\Delta X_{\max }$ and $R_{\mathrm{Had}}$ and the large number of events in the sample, the statistical significance of the corrections is large, greater than $5 \sigma_{\text {stat }}$ even for the combination of experimental systematic shifts within $1 \sigma_{\text {sys }}$ that are the most favorable for the models.
\end{abstract}

$37^{\text {th }}$ International Cosmic Ray Conference (ICRC 2021)

July 12th - 23rd, 2021

Online - Berlin, Germany

\footnotetext{
*Presenter
} 


\section{Introduction}

The mass composition of ultra-high energy cosmic rays (UHECR, above $10^{18} \mathrm{eV}$ ) is derived from properties of air showers that are initiated by interactions of UHECR with atmospheric nuclei. The air-shower property with high sensitivity to the mass of the primary UHECR particle is the depth of shower maximum $\left(X_{\max }\right)$. It can be inferred directly from the longitudinal profiles measured by the Fluorescence Detector (FD) telescopes such as those placed at the Pierre Auger Observatory [1]. The precision of inferences on the mass composition is limited by the systematic uncertainties in the properties of the hadronic interactions extrapolated from the accelerator data available at lower energies and in a limited volume of the phase space.

The consequent systematic uncertainties on the scale of simulated $\left\langle X_{\max }\right\rangle$ are difficult to estimate due to many factors influencing the hadronic interactions. From the difference of the Monte Carlo (MC) predictions using LHC-tuned hadronic interaction models (HI models) we can estimate that the uncertainty on the scale of $\left\langle X_{\max }\right\rangle$ is at least $30 \mathrm{~g} / \mathrm{cm}^{2}$ at ultra-high energies, which corresponds approximately to one-third of the difference between predictions for protons and iron nuclei, see the left panel of Fig. 1. The fluctuations of $X_{\max }$ differ by $\sim 5 \mathrm{~g} / \mathrm{cm}^{2}$ between different HI models.
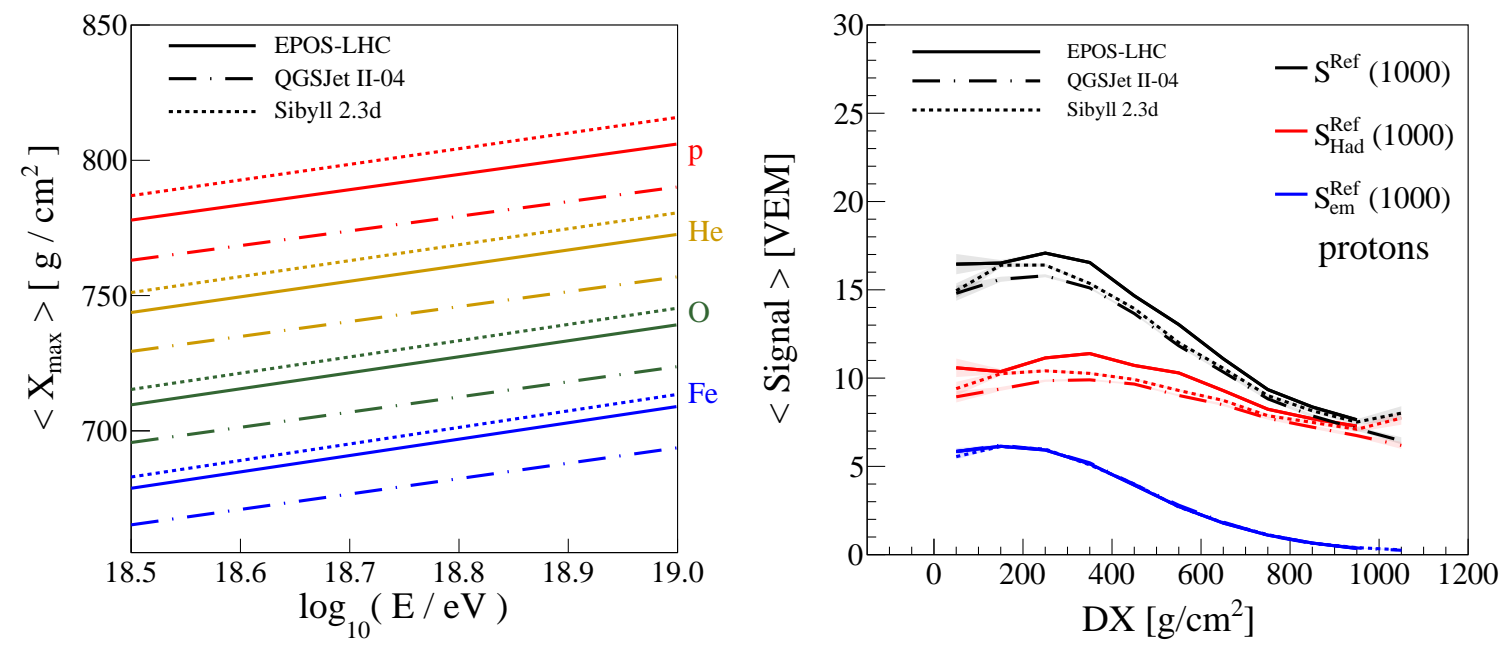

Figure 1: Left: the energy evolution of the mean of the $X_{\max }$ distribution predicted for three models of hadronic interactions and four primary species. Right: the dependence of average signal at ground level at $1000 \mathrm{~m}$ (black) on the distance of $X_{\max }$ to the ground $\left(D X=880 \mathrm{~g} / \mathrm{cm}^{2} / \cos (\theta)-X_{\max }\right)$ for proton showers. The hadronic and electromagnetic parts of the signal are shown in red and blue, respectively.

The signal at ground level at $1000 \mathrm{~m}$ from the core, $S(1000)$, measured by the water-Cherenkov stations of the Surface Detector (SD) at the Pierre Auger Observatory [2], is sensitive to the mass of the primary particle due to the large contribution of the muon component. However, various characteristics of air showers related to muons (signal normalization, arrival times) are not described well by HI models [3, 4]. As a result, inferences on the mass composition from the SD and FD data are in strong disagreement with each other. We divide $S(1000)$ into the hadronic part $S_{\mathrm{Had}}$ (including muons, electromagnetic halo from their decays, and local hadronic jets [5]) and electromagnetic part 
$S_{\mathrm{em}}$, see the right panel of Fig. 1. The predicted $S_{\mathrm{em}}$ (blue) is very universal, and the main differences in $S(1000)$ between the HI models come from the different normalization and attenuation of $S_{\mathrm{Had}}$.

\section{A method to adjust simulated $X_{\max }$ and hadronic signal at the ground}

We apply a slight modification of the method presented in [6] based on the maximum likelihood fit of the two-dimensional distributions of $X_{\max }$ and $S(1000)$ measured with the FD and SD of the Pierre Auger Observatory. The method aims to find which adjustments of the simulated $X_{\max }$ and $S(1000)$ are required for a consistent description of the measured two-dimensional $\left[X_{\max }, S(1000)\right]$ distributions.

The data are divided into five zenith-angle $(\theta)$ bins, as shown in Fig. 2. They are fitted with MC templates chosen in the form of a product of the Generalized Gumbel [7] (describing $X_{\max }^{\mathrm{Ref}}$ distributions) ${ }^{1}$ and Gaussian functions (describing $S^{\operatorname{Ref}}(1000)$ distributions) with the mean linearly dependent on $X_{\text {max }}^{\mathrm{Ref}}$.

In this work, we introduce ad-hoc adjustments in the fit to the simulated $\overline{X_{\max }^{\mathrm{Ref}}}$ and $\overline{S^{\operatorname{Ref}(1000)}}$ distributions, $\Delta X_{\max }$ and $f_{\mathrm{SD}}$, as

$$
\begin{aligned}
X_{\max }^{\operatorname{Ref}} & \equiv \widehat{X_{\max }^{\operatorname{Ref}}}+\Delta X_{\max }, \\
S^{\operatorname{Ref}}(1000) & \equiv S^{\overline{\operatorname{Ref}}(1000)} \cdot f_{\mathrm{SD}}(\theta),
\end{aligned}
$$

that are inspired by the universality properties of air showers. The rescaling parameter $f_{\mathrm{SD}}(\theta)$ is achieved through the multiplication of the scales of simulated hadronic signals at two extreme zenith-angle bins, $S_{\mathrm{Had}}\left(\theta_{\text {min }}\right) \cdot R_{\mathrm{Had}}\left(\theta_{\text {min }}\right)$ and $S_{\mathrm{Had}}\left(\theta_{\max }\right) \cdot R_{\mathrm{Had}}\left(\theta_{\text {max }}\right)$, leaving them as free fit parameters. $R_{\mathrm{Had}}(\theta)$ is linearly interpolated between $R_{\mathrm{Had}}\left(\theta_{\min }\right)$ and $R_{\mathrm{Had}}\left(\theta_{\max }\right)$ according to the average $D X=880 \mathrm{~g} / \mathrm{cm}^{2} / \cos \theta-X_{\max }$ value in the $\theta$ bin. The three separate corrections to the MC predictions result in the following scaling factor for the total simulated signal $S^{\operatorname{Ref}}(1000)$ considering the fraction of the hadronic signal $f_{\mathrm{Had}}=S_{\mathrm{Had}} / S(1000)$ :

$$
f_{\mathrm{SD}}(\theta)=R_{\mathrm{Had}}(\theta) \cdot g_{\mathrm{Had}}(\theta) \cdot \alpha_{\mathrm{Had}}(\theta) \cdot f_{\mathrm{Had}}(\theta)+g_{\mathrm{em}}(\theta) \cdot \alpha_{\mathrm{em}}(\theta) \cdot\left(1-f_{\mathrm{Had}}(\theta)\right),
$$

with factors stemming from the energy dependence of the signals, $\alpha_{\mathrm{Had}}=\frac{\left(E^{\mathrm{Ref}}\right)^{\beta-1 / B}}{\left\langle E_{\mathrm{FD}}^{\beta-1 / B}\right\rangle(\theta)}$ and $\alpha_{\mathrm{em}}=$ $\frac{\left(E^{\mathrm{Ref}}\right)^{1-1 / B}}{\left\langle E_{\mathrm{FD}}^{1-1 / B}\right\rangle(\theta)}$, where the parameter $\beta=0.92$ was chosen in accordance with [8], and $B=1.031$ is the $\mathrm{SD}$ energy calibration parameter [9]. We take into account the changes in the signal arising due to the adjustment of $X_{\max }$. The parameterizations, $g_{\text {Had }}(\theta)$ and $g_{\mathrm{em}}(\theta)$, of the dependence of $\left\langle S_{\text {Had }}\right\rangle$ and $\left\langle S_{\mathrm{em}}\right\rangle$ on the distance of $X_{\max }$ to the ground, respectively, are used for this purpose considering the adjustment of the $S_{\mathrm{Had}}$ attenuation as well. For instance, the change of $S(1000)$ reaches $\sim 7 \%$ at most in case of $\Delta X_{\max } \sim 50 \mathrm{~g} / \mathrm{cm}^{2}$.

The MC templates consist of a sum of templates for individual primary species weighted by their relative fractions $f_{i}, i=$ proton $(\mathrm{p})$, helium $(\mathrm{He})$, oxygen $(\mathrm{O})$, iron $(\mathrm{Fe})$, which serve as other three free fit parameters $\left(\sum f_{\mathrm{i}}=1\right)$. This way, the result of the fit is the combination of four primary particles (p, He, $\mathrm{O}, \mathrm{Fe}$ ) and of the adjustment factors $\Delta X_{\max }, R_{\mathrm{Had}}\left(\theta_{\min }\right)$ and $R_{\mathrm{Had}}\left(\theta_{\max }\right)$ for which the best description of the measured $\left[X_{\max }, S(1000)\right]$ distributions is achieved.

\footnotetext{
1'Ref' indicates that we use the observables scaled to the reference energy $E^{\text {Ref }}=10^{18.7} \mathrm{eV}$.
} 


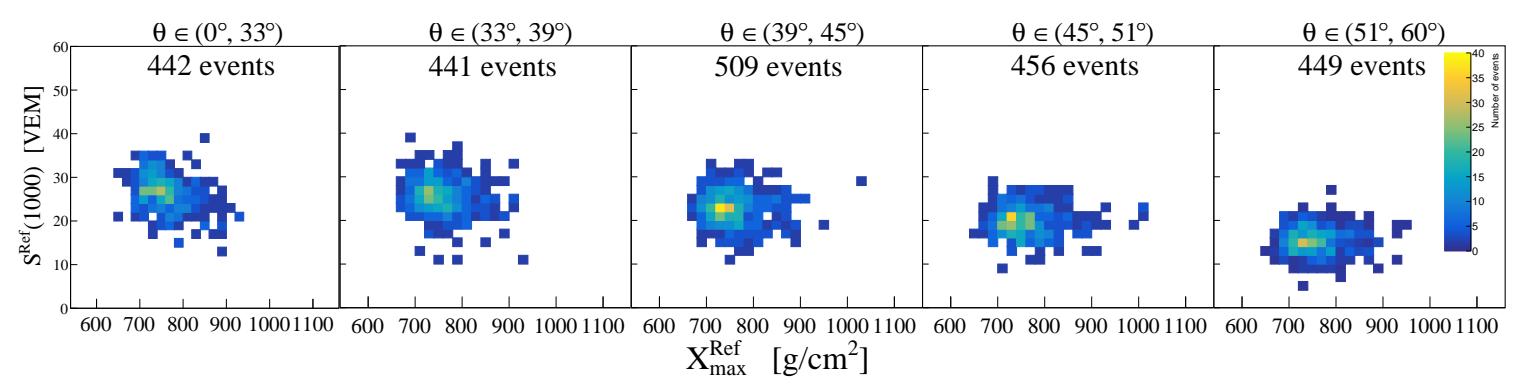

Figure 2: Two-dimensional distributions of $S(1000)^{\operatorname{Ref}}$ and $X_{\max }^{\mathrm{Ref}}$ for data of the Pierre Auger Observatory measured in the energy range $10^{18.5}-10^{19.0} \mathrm{eV}$ in five zenith-angle bins.

The correlation between $X_{\max }$ and $S(1000)$, governed by the general properties of air showers and thus weakly dependent on characteristics of particular HI models [10], is implicitly accounted for in the fits helping to reduce the degeneracy between the mass composition and the scale of simulated $\left\langle X_{\max }\right\rangle$. In the absence of differences other than the main ones between HI models and data in $\Delta X_{\max }, R_{\mathrm{Had}}\left(\theta_{\min }\right)$ and $R_{\mathrm{Had}}\left(\theta_{\max }\right)$, the fit would result in totally model-independent inferences on the mass composition. Clearly, this is not the case, and there are remaining higherorder differences not taken into account in the current method, such as differences between HI models in the widths of $X_{\max }$ distributions, separations in $X_{\max }$ between the primary species, and mass dependencies of $R_{\mathrm{Had}}\left(\theta_{\min }\right)$ and $R_{\mathrm{Had}}\left(\theta_{\max }\right)$ etc.

\section{Data and simulations}

We use the events detected at the same time by SD and FD of the Pierre Auger Observatory during the period 1/1/2004 - 31/12/2018. The range of the FD energies is $10^{18.5}-10^{19.0} \mathrm{eV}$ (mean energy $\sim 10^{18.7} \mathrm{eV}$ ), with the lower limit corresponding to the $100 \%$ efficiency of the SD for zenith angles below 60 degrees. The FD selection is the same as used for the $X_{\max }$ analysis $[11,12]$ and the SD selection follows that of the SD energy-spectrum analysis [9]. In total, 2297 high-quality events were selected for the analysis (see Fig. 2).

The simulated air showers were produced using CORSIKA 7.7400 [13] and the detector simula-

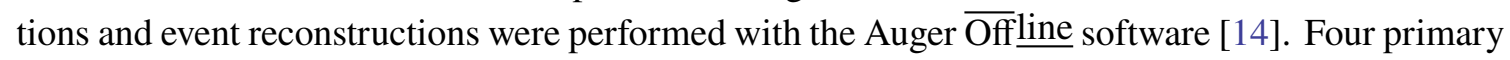
particles (p, He, O, Fe) and three HI models: Epos-LhC [15], QgSJet II-04 [16] and Sibyll 2.3d [17] were used.

\section{Results}

The examples of description of projected $S^{\text {Ref }}(1000)$ distributions at two extreme $\theta$-bins and of the projected $X_{\max }^{\mathrm{Ref}}$ distribution are shown in Fig. 3c together with the $\theta$ evolution of the GideonHollister correlation coefficient $\left(r_{\mathrm{G}}\right)[18]$ of the $\left[X_{\max }, S(1000)\right]$ distributions. The lowest negative logarithm of the likelihood ratio $(\mathcal{L})$ was found to be $\sim 480$ ( $p$-value $\simeq 2.6 \%$ ) for Epos-LHC, $\sim 507$ $(p$-value $\simeq 3.6 \%)$ for QGSJet II-04, and $\sim 478$ ( $p$-value $\simeq 18 \%)$ for SibyLL $2.3 \mathrm{~d}$. To illustrate the improvement of the data description introducing the adjustment of the simulated $X_{\max }$, we show the same comparison in Fig. $3 \mathrm{~b}$ for $\Delta X_{\max }=0 \mathrm{~g} / \mathrm{cm}^{2}$. The data description without any adjustment to MC predictions is shown in Fig. 3 a with mass composition obtained from the $X_{\max }$ fit. 

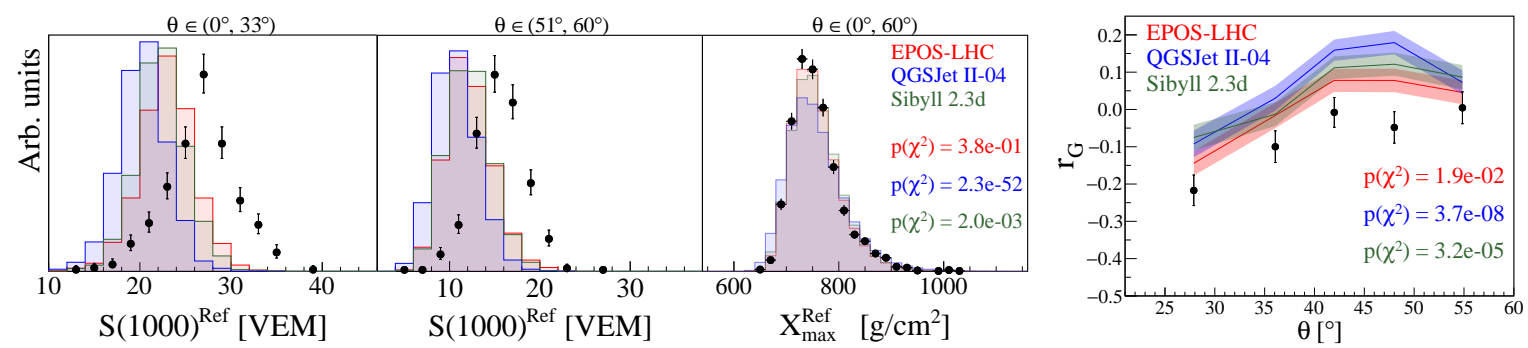

(a) No MC corrections
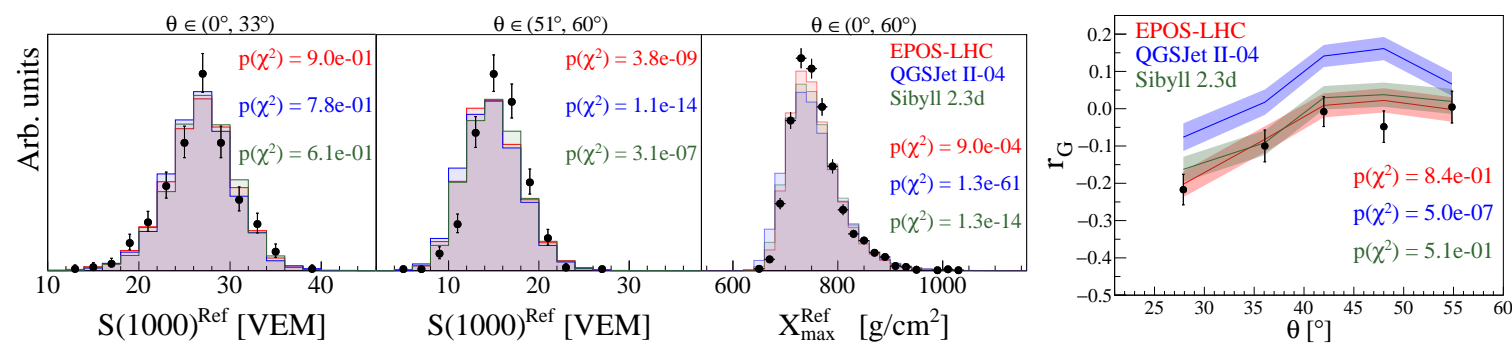

(b) MC corrections: $R_{\mathrm{Had}}(\theta)$
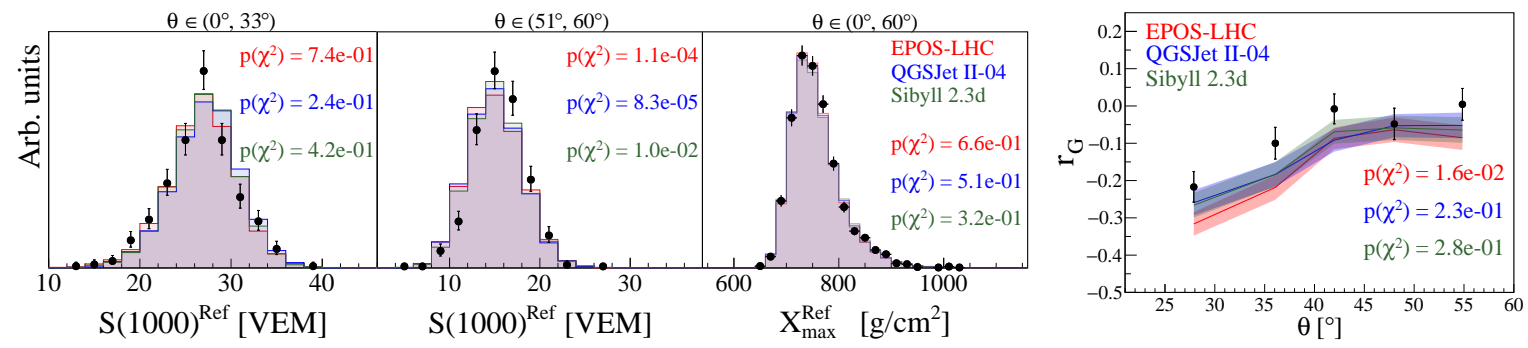

(c) MC corrections: $\Delta X_{\max }$ and $R_{\mathrm{Had}}(\theta)$

Figure 3: From left: $S(1000)^{\text {Ref }}$ distributions in two extreme zenith-angle bins, the $X_{\max }^{\text {Ref }}$ distribution and the $r_{\mathrm{G}}$ correlation parameter of $\left[X_{\max }, S(1000)\right]$ as a function of the zenith angle. Top (a): results of the $X_{\max }^{\mathrm{Ref}}$ fit; middle(b): results of the fit with $\Delta X_{\max }$ fixed to zero $\mathrm{g} / \mathrm{cm}^{2}$; bottom (c): results of the full fit.

The resulting rescaling parameters of the simulated hadronic signal $R_{\mathrm{Had}}\left(\theta_{\min }\right)$ and $R_{\mathrm{Had}}\left(\theta_{\max }\right)$ are shown in the left panel of Fig. 4. We found that the adjustment of the attenuation of $S_{\mathrm{Had}}$ (difference between $R_{\text {Had }}\left(\theta_{\min }\right)$ and $R_{\text {Had }}\left(\theta_{\max }\right)$ ) depends mainly on the experimental energy scale, see the right panel of Fig. 6. For the energy scale currently adopted at the Pierre Auger Observatory, the fit results prefer the attenuation of $S_{\text {Had }}$ predicted by Epos-LHC. For all three HI models, a deeper $X_{\max }$ prediction is preferred with $\Delta X_{\max }$ values equal to $22 \pm 3_{-11}^{+14} \mathrm{~g} / \mathrm{cm}^{2}$ for Epos-LHC, $48 \pm 2_{-12}^{+9} \mathrm{~g} / \mathrm{cm}^{2}$ for QgSJet II-04, and $30 \pm 2_{-15}^{+9} \mathrm{~g} / \mathrm{cm}^{2}$ for Sibyll 2.3d, see Fig. 5. Such shifts of simulated $X_{\max }$ values lead to a heavier mass composition (right panel of Fig. 4) compared to the inferences with the unaltered $\mathrm{HI}$ models. As expected, the inferences on the mass composition are now much less model-dependent.

The increase of the MC prediction on $X_{\max }$, resulting in the increase of the signal at the ground, alleviates the problem with the deficit of muons in the predictions of HI models, as, e.g., in [4]. Still, for a satisfactory description of the data, the hadronic signal in $\mathrm{HI}$ models should be increased by $15 \pm 2_{-16}^{+20} \%$ for Epos-LHC, by $24 \pm 2_{-19}^{+23} \%$ for QGSJET II-04, and by $17 \pm 2_{-17}^{+22} \%$ for SibYLL $2.3 \mathrm{~d}$ 

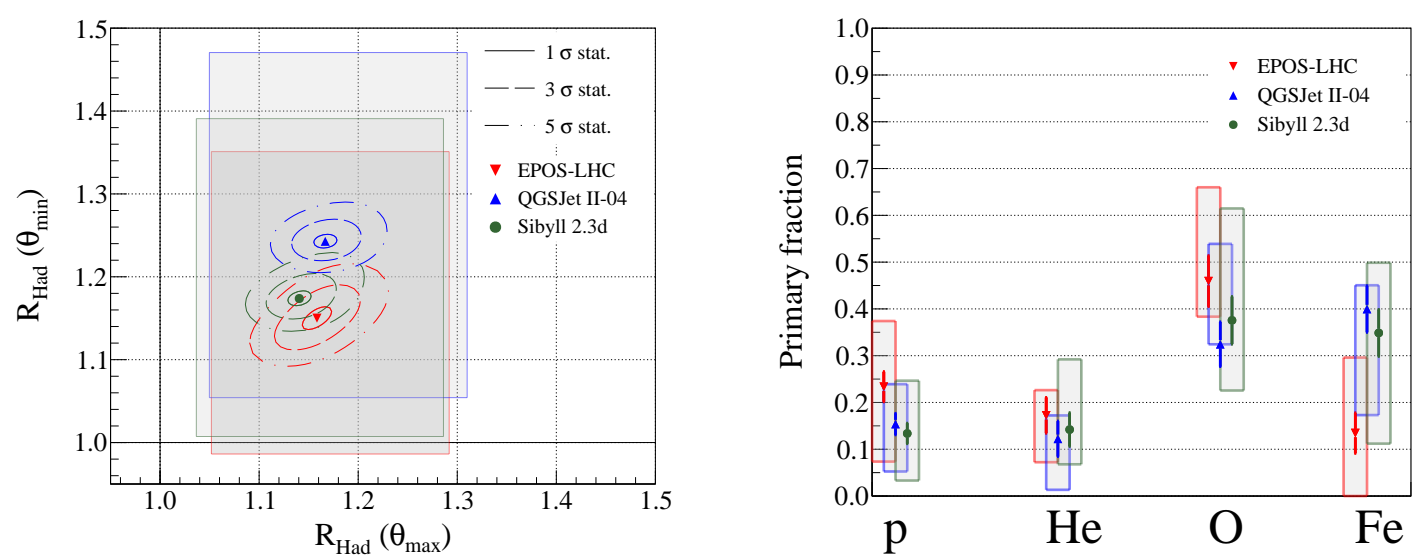

Figure 4: Results of the fits of $\left[X_{\max }, S(1000)\right]$ distributions for Epos-LHC, QGSJET II-04 and SIBYLL 2.3d. Left: the rescaling parameters for the hadronic signal. The contours denote regions with 1,3 and $5 \sigma$ statistical uncertainties. Right: the fractions of primary nuclei. The gray bands in both panels indicate the size of the total systematic uncertainties.
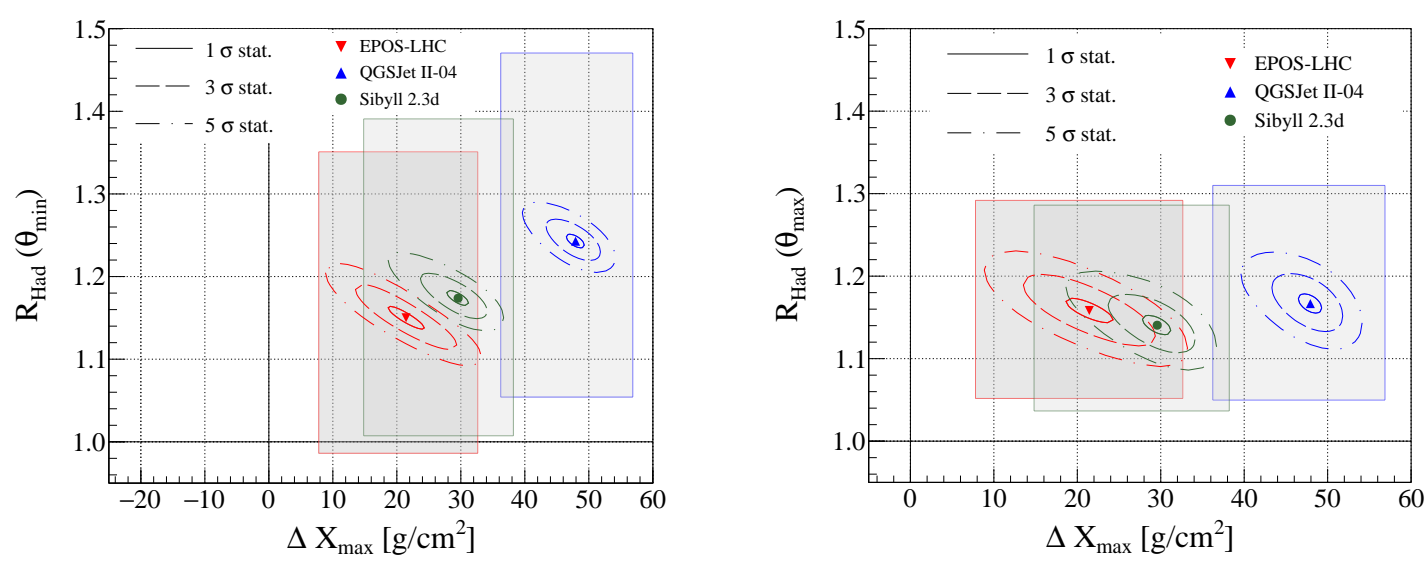

Figure 5: Correlations between the rescaling parameters of the hadronic signal at the minimal (left) and maximal (right) zenith angles and the shift of $X_{\max }$ scale. The contours mark 1, 3, and $5 \sigma$ statistical errors. The gray bands indicate the size of total systematic uncertainties.

at low zenith angles of the showers $\left(\sim 28^{\circ}\right)$. For high zenith angles $\left(\sim 55^{\circ}\right)$, the hadronic signal at ground should be increased by $16 \pm 2_{-11}^{+13} \%$ for EPOS-LHC, by $17 \pm 2_{-12}^{+9} \%$ for QGSJET II-04, and by $14 \pm 1_{-10}^{+15} \%$ for SiBYLL $2.3 \mathrm{~d}$.

\subsection{Systematic uncertainties}

There are four dominant sources of systematic uncertainties influencing the results. Three of them are $1 \sigma_{\text {sys }}$ experimental uncertainties on the energy scale $\pm 14 \%$ [9], $X_{\max }^{+8} \mathrm{~g} / \mathrm{cm}^{2}$ [11] and $S(1000) \pm 5 \%$ [2]. The fourth source of systematic uncertainty is related to the biases of the method itself, as estimated from MC-MC studies $\left(_{-4}^{+2} \mathrm{~g} / \mathrm{cm}^{2}\right.$ for $\Delta X_{\max },{ }_{-3}^{+1} \%$ for $R_{\mathrm{Had}}\left(\theta_{\min }\right)$, and $\pm 1 \%$ for $R_{\text {Had }}\left(\theta_{\max }\right)$ ). All four uncertainties are summed in quadrature, and the total systematic uncertainties are shown by gray bands in Figs. 4 and 5 . 
For each possible combination of the three (positive and negative) $1 \sigma_{\text {sys }}$ systematic experimental uncertainties, we calculated the statistical significance $\sigma_{\text {stat }}$ of MC corrections as the Cartesian distance in three-dimensional space of the three MC corrections in units of their statistical errors. This way we explore systematic experimental uncertainties $1 \sigma_{\text {sys }}$ to see if they can conspire to evade the need to adjust model predictions. However, due to the analysis relying on the correlations between the signal at ground level, zenith angle, and $X_{\max }$, whereas the systematics are largely uncorrelated, the result is robust. Employing systematic shifts within $1 \sigma_{\text {sys }}$ to reduce the adjustments in $X_{\max }$ and $R_{\mathrm{Had}}$ as much as possible, the needed adjustments of model predictions are greater than $5 \sigma_{\text {stat }}$ for all models.
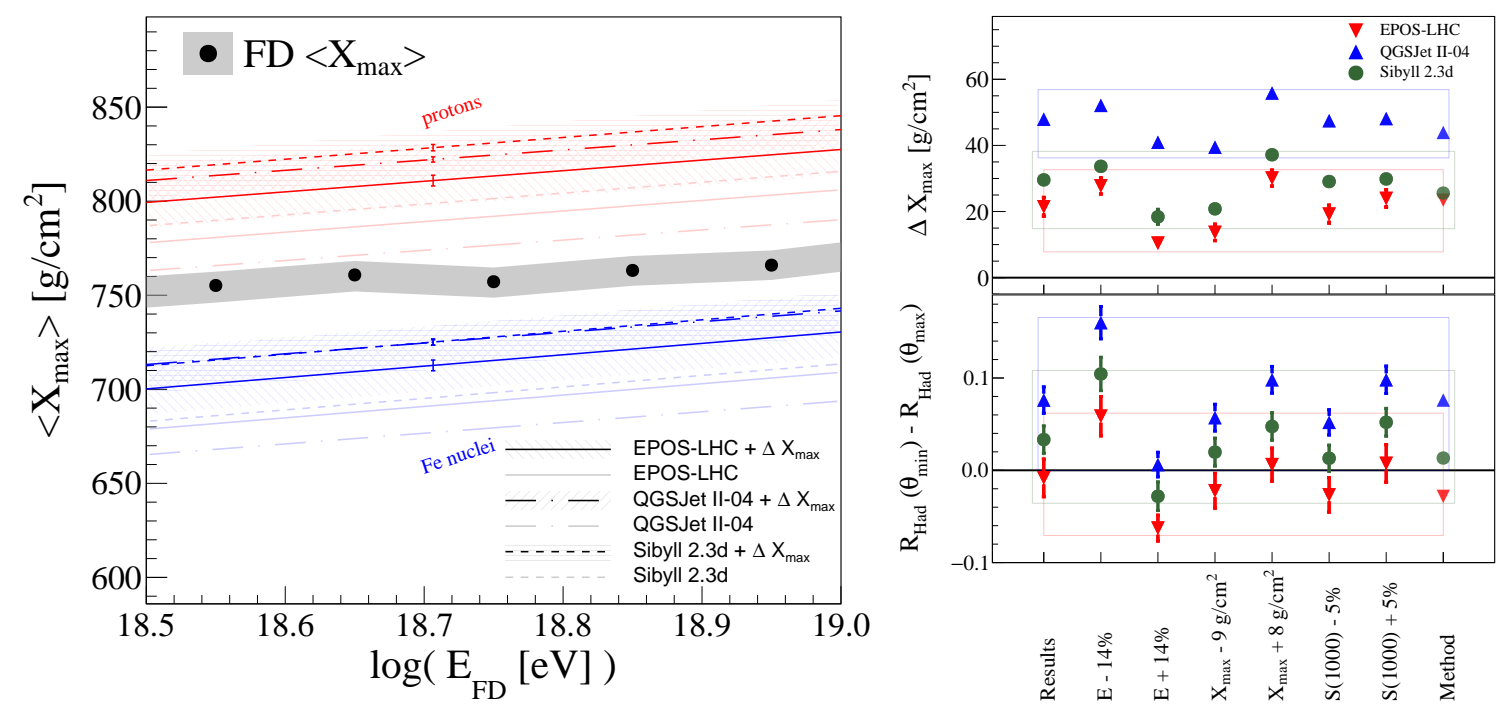

Figure 6: Left: the energy evolution of the mean $X_{\max }$ measured at the Pierre Auger Observatory using FD [12]. The adjusted MC predictions on $\left\langle X_{\max }\right\rangle$ obtained in this work are shown in red and blue for protons and iron nuclei, respectively. The bands correspond to the systematic uncertainties. The lighter color lines indicate unmodified MC predictions. Right: the best fit results on the $\Delta X_{\max }$ and correction to the $S_{\mathrm{Had}}$ attenuation for the individual systematic effects. The bands illustrate the total systematic uncertainty summed in quadrature.

\section{Discussion}

On the left panel of Fig. 6, we show the adjusted predictions of $\left\langle X_{\max }\right\rangle$ for protons and iron nuclei together with the measurements at the Pierre Auger Observatory with the FD [12]. By artificially smearing $X_{\max }$ in the case of EPOS-LHC, we checked that the main difference between the adjusted model predictions on $\left\langle X_{\max }\right\rangle$ between EPOS-LHC and the other two models is due to the narrower $X_{\max }$ distributions predicted by EPOS-LHC. From the current analysis, it follows that the hadronic signal at $1000 \mathrm{~m}$ from the shower core should be increased by $\sim 15-25 \%$ for $\theta \in\left(0^{\circ}, 33^{\circ}\right)$ $\left(D X \sim 240 \mathrm{~g} / \mathrm{cm}^{2}\right)$ and by $\sim 15 \%$ for $\theta \in\left(51^{\circ}, 60^{\circ}\right)\left(D X \sim 780 \mathrm{~g} / \mathrm{cm}^{2}\right)$ for all three HI models. This increase is smaller compared to our earlier findings [4], but this is mainly because, in the current method, adjustments are applied not only to the hadronic signal but also to $X_{\max }$. The adjusted attenuation of the hadronic component is correlated with the change of the energy scale, 
with the one currently adopted at the Pierre Auger Observatory preferring the attenuation predicted by EPOS-LHC, see the right panel of Fig. 6.

In summary, we presented a novel method allowing one to infer deficiencies in the HI models in the description of both longitudinal and lateral development of air showers, accounting for which naturally leads to the reduction of the systematic uncertainties in the inferences on the mass composition. We use a global fit of two-dimensional distributions of $X_{\max }$ and $S(1000)$ measured with the FD and SD of the Pierre Auger Observatory at five different zenith angles. This way, we fit mass composition and ad-hoc adjustments of predictions of $\mathrm{HI}$ models in the energy range $10^{18.5}-10^{19.0} \mathrm{eV}$. In this work, we have left as free parameters an overall shift in the predicted $X_{\max }$ and a scale of the hadronic component at two extreme zenith angles. An overall improvement in the description of measured data of $S^{\mathrm{Ref}}(1000), X_{\max }^{\mathrm{Ref}}$, and their correlation was achieved for all three HI models. These models are shifted towards deeper values and, consequently, the deficit in the simulated hadronic signal is alleviated with respect to the previous studies that did not consider an adjustment in the predicted $\left\langle X_{\max }\right\rangle$. Without the three MC adjustments, the HI models are found to be at variance with the data with a significance $\gtrsim 5 \sigma_{\text {stat }}$ accounting for shifts of $\lesssim 1 \sigma_{\text {sys }}$. We should note that we have taken into account only the simplest but leading ad-hoc adjustments to the predicted $X_{\max }$ and $S_{\mathrm{Had}}(\theta)$. Accounting for other possible adjustments, like, e.g., $X_{\max }$ and $S(1000)$ fluctuations, and their impact on the predictions mentioned above, will be further investigated.

\section{Acknowledgements}

This work is funded by the Czech Republic grants of MEYS CR: LTT18004, LM2015038, LM2018102, CZ.02.1.01/0.0/0.0/16_013/0001402, CZ.02.1.01/0.0/0.0/18_046/0016010.

\section{References}

[1] A. Aab et al. [Pierre Auger Coll.], Nucl. Instrum. Meth. A 798 (2015) 172.

[2] A. Aab et al. [Pierre Auger Coll.], J. Instrum. 15 (2020) P10021.

[3] A. Aab et al. [Pierre Auger Coll.], Phys. Rev. D 91 (2015) 032003. A. Aab et al. [Pierre Auger Coll.], Phys. Rev. D 92 (2015) 019903. A. Aab et al. [Pierre Auger Coll.], Eur. Phys. J. C 80 (2020) 751. A. Aab et al. [Pierre Auger Coll.], Phys. Rev. Lett. 126 (2021) 152002. A. Aab et al. [Pierre Auger Coll.], Phys. Rev. D 96 (2017) 122003.

[4] A. Aab et al. [Pierre Auger Coll.], Phys. Rev. Lett. 117 (2016) 192001.

[5] M. Ave et al., Astropart. Phys. 87 (2017) 23. M. Ave et al., Astropart. Phys. 88 (2017) 46.

[6] J. Vícha et al., Proc. 36th Int. Cosmic Ray Conf., Madison, USA (2019), PoS(ICRC2019)452.

[7] M. De Domenico et al., J. Cosmol. Astropart. P. 07 (2013) 050.

[8] K.-H. Kampert \& M. Unger, Astropart. Phys. 35 (2012) 660.

[9] A. Aab et al. [Pierre Auger Coll.], Phys. Rev. D 102 (2020) 062005.

[10] A. Aab et al. [Pierre Auger Coll.], Phys. Lett. B. 762 (2016) 288.

[11] A. Aab et al. [Pierre Auger Coll.], Phys. Rev. D 90 (2014) 122005.

[12] A. Yushkov for the Pierre Auger Collaboration, Proc. 36th Int. Cosmic Ray Conf., Madison, USA (2019), PoS(ICRC19)482.

[13] D. Heck et al., Report FZKA Forschungszentrum Karlsruhe 6019 (1998).

[14] S. Argiro et al., Nucl. Instrum. Meth. A 580 (2007) 1485.

[15] T. Pierog et al., Phys. Rev. C 92 (2015) 034906.

[16] S. Ostapchenko, Phys. Rev. D 83 (2011) 014018.

[17] F. Riehn et al., Phys. Rev. D 102 (2020) 063002.

[18] R. Gideon \& R. Hollister, J. Am. Stat. Assoc. 82 (1987) 656. 


\section{The Pierre Auger Collaboration}

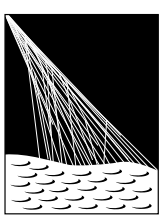

PIERRE

AUSGERVATORY

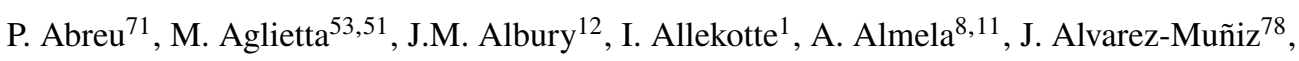
R. Alves Batista ${ }^{79}$, G.A. Anastasi ${ }^{62,51}$, L. Anchordoqui ${ }^{86}$, B. Andrada ${ }^{8}$, S. Andringa ${ }^{71}$, C. $\mathrm{Aramo}^{49}$, P.R. Araújo Ferreira ${ }^{41}$, J. C. Arteaga Velázquez ${ }^{66}$, H. Asorey $^{8}$, P. Assis ${ }^{71}$, G. Avila ${ }^{10}$, A.M. Badescu ${ }^{74}$, A. Bakalova ${ }^{31}$, A. Balaceanu ${ }^{72}$, F. Barbato ${ }^{44,45}$, R.J. Barreira Luz $^{71}$, K.H. Becker ${ }^{37}$, J.A. Bellido ${ }^{12,68}$, C. Berat ${ }^{35}$, M.E. Bertaina ${ }^{62,51}$, X. Bertou ${ }^{1}$, P.L. Biermann ${ }^{b}$, V. Binet ${ }^{6}$, K. Bismark ${ }^{38,8}$, T. Bister ${ }^{41}$, J. Biteau ${ }^{36}$, J. Blazek ${ }^{31}$, C. Bleve ${ }^{35}$, M. Boháčová ${ }^{31}$, D. Boncioli ${ }^{56,45}$, C. Bonifazi ${ }^{25}$, L. Bonneau Arbeletche ${ }^{20}$, N. Borodai ${ }^{69}$, A.M. Botti ${ }^{8}$, J. Brack ${ }^{d}$, T. Bretz ${ }^{41}$, P.G. Brichetto Orchera ${ }^{8}$, F.L. Briechle ${ }^{41}$, P. Buchholz ${ }^{43}$, A. Bueno ${ }^{77}$, S. Buitink ${ }^{14}$, M. Buscemi ${ }^{46}$, M. Büsken ${ }^{38,8}$, K.S. Caballero-Mora ${ }^{65}$, L. Caccianiga ${ }^{58,48}$, F. Canfora ${ }^{79,80}$, I. Caracas ${ }^{37}$, J.M. Carceller ${ }^{77}$, R. Caruso ${ }^{57,46}$, A. Castellina ${ }^{53,51}$, F. Catalani ${ }^{18}$, G. Cataldi ${ }^{47}$, L. Cazon ${ }^{71}$, M. Cerda ${ }^{9}$, J.A. Chinellato ${ }^{21}$, J. Chudoba ${ }^{31}$, L. Chytka ${ }^{32}$, R.W. Clay ${ }^{12}$, A.C. Cobos Cerutti ${ }^{7}$, R. Colalillo ${ }^{59,49}$, A. Coleman ${ }^{92}$, M.R. Coluccia ${ }^{47}$, R. Conceição ${ }^{71}$, A. Condorelli ${ }^{44,45}$, G. Consolati ${ }^{48,54}$, F. Contreras ${ }^{10}$, F. Convenga ${ }^{55,47}$, D. Correia dos $\operatorname{Santos}^{27}$, C.E. Covault ${ }^{84}$, S. Dasso ${ }^{5,3}$, K. Daumiller ${ }^{40}$, B.R. Dawson ${ }^{12}$, J.A. Day ${ }^{12}$, R.M. de Almeida ${ }^{27}$, J. de Jesús ${ }^{8,40}$, S.J. de Jong ${ }^{79,80}$, G. De Mauro ${ }^{79,80}$, J.R.T. de Mello Neto ${ }^{25,26}$, I. De Mitrii ${ }^{44,45}$, J. de Oliveira ${ }^{17}$, D. de Oliveira Franco ${ }^{21}$, F. de Palma ${ }^{55,47}$, V. de Souza ${ }^{19}$, E. De Vito ${ }^{55,47}$, M. del Río ${ }^{10}$, O. Deligny ${ }^{33}$, L. Deval ${ }^{40}$, A. di Matteo ${ }^{51}$, C. Dobrigkeit ${ }^{21}$, J.C. D'Olivo ${ }^{67}$, L.M. Domingues Mendes ${ }^{71}$, R.C. dos Anjos ${ }^{24}$, D. dos $\operatorname{Santos}^{27}$, M.T. Dova ${ }^{4}$, J. $\mathrm{Ebr}^{31}$, R. Enge ${ }^{38,40}$, I. Epicoco ${ }^{55,47}$, M. Erdmann ${ }^{41}$, C.O. Escobar ${ }^{a}$, A. Etchegoyen ${ }^{8,11}$, H. Falcke ${ }^{79,81,80}$, J. Farmer ${ }^{91}$, G. Farrar ${ }^{89}$, A.C. Fauth ${ }^{21}$, N. Fazzini ${ }^{a}$, F. Feldbusch ${ }^{39}$, F. Fenu ${ }^{53,51}$, B. Fick ${ }^{88}$, J.M. Figueira ${ }^{8}$, A. Filipčičç ${ }^{6,75}$, T. Fitoussi ${ }^{40}$, T. Fodran ${ }^{79}$, M.M. Freire ${ }^{6}$, T. Fujii ${ }^{91, e}$, A. Fuster ${ }^{8,11}$, C. Galea ${ }^{79}$, C. Galelli ${ }^{58,48}$, B. García ${ }^{7}$, A.L. Garcia Vegas ${ }^{41}$, H. Gemmeke ${ }^{39}$, F. Gesualdi ${ }^{8,40}$, A. Gherghel-Lascu ${ }^{72}$, P.L. Ghia ${ }^{33}$, U. Giaccari ${ }^{79}$, M. Giammarchi ${ }^{48}$, J. Glombitza ${ }^{41}$, F. Gobbi ${ }^{9}$, F. Gollan ${ }^{8}$, G. Golup ${ }^{1}$, M. Gómez Berisso ${ }^{1}$, P.F. Gómez Vitale ${ }^{10}$, J.P. Gongora ${ }^{10}$, J.M. González ${ }^{1}$, N. González ${ }^{13}$, I. Goos ${ }^{1,40}$, D. Góra ${ }^{69}$, A. Gorgi ${ }^{33,51}$, M. Gottowik ${ }^{37}$, T.D. Grubb ${ }^{12}$, F. Guarino ${ }^{59,49}$, G.P. Guedes ${ }^{22}$, E. Guido ${ }^{51,62}$, S. $\mathrm{Hahn}^{40,8}$, P. Hamal ${ }^{31}$, M.R. Hampel ${ }^{8}$, P. Hansen ${ }^{4}$, D. Harari ${ }^{1}$, V.M. Harvey ${ }^{12}$, A. Haungs ${ }^{40}$, T. Hebbeker ${ }^{41}$, D. Heck ${ }^{40}$, G.C. Hill ${ }^{12}$, C. Hojvat ${ }^{a}$, J.R. Hörandel ${ }^{79,80}$, P. Horvath ${ }^{32}$, M. Hrabovský ${ }^{32}$, T. Huege ${ }^{40,14}$, A. Insolia ${ }^{57,46}$, P.G. Isar ${ }^{73}$, P. Janecek ${ }^{31}$, J.A. Johnsen ${ }^{85}$, J. Jurysek ${ }^{31}$, A. Kääpä ${ }^{37}$, K.H. Kampert ${ }^{37}$, N. Karastathis ${ }^{40}$, B. Keilhauer ${ }^{40}$, J. Kemp ${ }^{41}$, A. Khakurdikar ${ }^{79}$, V.V. Kizakke Covilakam ${ }^{8,40}$, H.O. Klages ${ }^{40}$, M. Kleifges ${ }^{39}$, J. Kleinfeller ${ }^{9}$, M. Köpke ${ }^{38}$, N. Kunka ${ }^{39}$, B.L. Lago ${ }^{16}$, R.G. Lang ${ }^{19}$, N. Langner ${ }^{41}$, M.A. Leigui de Oliveira ${ }^{23}$, V. Lenok ${ }^{40}$, A. Letessier-Selvon ${ }^{34}$, I. Lhenry-Yvon ${ }^{33}$, D. Lo Presti $^{57,46}$, L. Lopes ${ }^{71}$, R. López ${ }^{63}$, L. Lu ${ }^{93}$, Q. Luce ${ }^{38}$, J.P. Lundquist ${ }^{75}$, A. Machado Payeras ${ }^{21}$, G. Mancarella $a^{5,47}$, D. Mandat ${ }^{31}$, B.C. Manning ${ }^{12}$, J. Manshanden ${ }^{42}$, P. Mantsch ${ }^{a}$, S. Marafico ${ }^{33}$, A.G. Mariazzi ${ }^{4}$, I.C. Mariş ${ }^{13}$, G. Marsella ${ }^{60,46}$, D. Martello ${ }^{55,47}$, S. Martinelli ${ }^{40,8}$, O. Martínez Bravo ${ }^{63}$, M. Mastrodicasa ${ }^{56,45}$, H.J. Mathes ${ }^{40}$, J. Matthews ${ }^{87}$, G. Matthiae ${ }^{61,50}$, E. Mayotte ${ }^{37}$, P.O. Mazur ${ }^{a}$, G. MedinaTanco $^{67}$, D. Melo ${ }^{8}$, A. Menshikov ${ }^{39}$, K.-D. Merenda ${ }^{85}$, S. Michal ${ }^{32}$, M.I. Micheletti ${ }^{6}$, L. Miramonti ${ }^{58,48}$, S. Mollerach ${ }^{1}$, F. Montanet ${ }^{35}$, C. Morello ${ }^{53,51}$, M. Mostafá ${ }^{90}$, A.L. Müller ${ }^{8}$, M.A. Muller ${ }^{21}$, K. Mulrey ${ }^{14}$, R. Mussa ${ }^{51}$, M. Muzio ${ }^{89}$, W.M. Namasaka ${ }^{37}$, A. Nasr-Esfahani ${ }^{37}$, L. Nellen ${ }^{67}$, M. Niculescu-Oglinzanu ${ }^{72}$, M. Niechciol ${ }^{43}$, D. Nitz ${ }^{88}$, D. Nosek ${ }^{30}$, V. Novotny ${ }^{30}$, L. Nožka ${ }^{32}$, A Nucita ${ }^{55,47}$, L.A. Núñez ${ }^{29}$, M. Palatka ${ }^{31}$, J. Pallotta ${ }^{2}$, P. Papenbreer ${ }^{37}$, G. Parente ${ }^{78}$, A. Parra ${ }^{63}$, J. Pawlowsky ${ }^{37}$, M. Pech ${ }^{31}$, F. Pedreira ${ }^{78}$, J. Pȩkala ${ }^{69}$, R. Pelayo ${ }^{64}$, J. Peña-Rodriguez ${ }^{29}$, E.E. Pereira Martins ${ }^{38,8}$, J. Perez Armand ${ }^{20}$, C. Pérez Bertolli $^{8,40}$, M. Perlin ${ }^{8,40}$, L. Perrone ${ }^{55,47}$, S. Petrera ${ }^{44,45}$, T. Pierog ${ }^{40}$, M. Pimenta ${ }^{71}$, V. Pirronello ${ }^{57,46}$, M. Platino ${ }^{8}$, B. Pont $^{79}$, M. Pothast ${ }^{80,79}$, P. Privitera ${ }^{91}$, M. Prouza ${ }^{31}$, A. Puyleart ${ }^{88}$, S. Querchfeld ${ }^{37}$, J. Rautenberg ${ }^{37}$, D. Ravignani ${ }^{8}$, M. Reininghaus ${ }^{40,8}$, J. Ridky ${ }^{31}$, F. Riehn ${ }^{71}$, M. Risse ${ }^{43}$, V. Rizi ${ }^{56,45}$, W. Rodrigues de Carvalho ${ }^{20}$, J. Rodriguez Rojo ${ }^{10}$, M.J. Roncoroni ${ }^{8}$, S. Rossoni ${ }^{42}$, M. Roth ${ }^{40}$, E. Roulet ${ }^{1}$, A.C. Rovero ${ }^{5}$, P. Ruehl ${ }^{43}$, S.J. Saffi ${ }^{12}$, A. Saftoiu ${ }^{72}$, F. Salamida ${ }^{56,45}$, H. Salazar ${ }^{63}$, G. Salina ${ }^{50}$, J.D. Sanabria Gomez $^{29}$, F. Sánchez ${ }^{8}$, E.M. Santos ${ }^{20}$, E. Santos ${ }^{31}$, F. Sarazin ${ }^{85}$, R. Sarmento ${ }^{71}$, C. Sarmiento-Cano ${ }^{8}$, 
R. Sato ${ }^{10}$, P. Savina ${ }^{55,47,33}$, C.M. Schäfer ${ }^{40}$, V. Scherini ${ }^{47}$, H. Schieler ${ }^{40}$, M. Schimassek ${ }^{38,8}$, M. Schimp ${ }^{37}$, F. Schlüter ${ }^{40,8}$, D. Schmidt ${ }^{38}$, O. Scholten ${ }^{83,14}$, P. Schovánek ${ }^{31}$, F.G. Schröder ${ }^{92,40}$, S. Schröder ${ }^{37}$, J. Schulte ${ }^{41}$, S.J. Sciutto ${ }^{4}$, M. Scornavacche ${ }^{8,40}$, A. Segreto ${ }^{52,46}$, S. Sehgal ${ }^{37}$, R.C. Shellard ${ }^{15}$, G. Sigl ${ }^{42}$, G. Silli ${ }^{8,40}$, O. Sima ${ }^{72, f}$, R. Šmída ${ }^{91}$, P. Sommers ${ }^{90}$, J.F. Soriano ${ }^{86}$, J. Souchard ${ }^{35}$, R. Squartini ${ }^{9}$, M. Stadelmaier ${ }^{40,8}$, D. Stanca ${ }^{72}$, S. Stanič ${ }^{75}$, J. Stasielak ${ }^{69}$, P. Stassi ${ }^{35}$, A. Streich ${ }^{38,8}$, M. Suárez-Durán ${ }^{13}$, T. Sudholz ${ }^{12}$, T. Suomijärvi ${ }^{36}$, A.D. Supanitsky ${ }^{8}$, Z. Szadkowski ${ }^{70}$, A. Tapia ${ }^{28}$, C. Taricco ${ }^{62,51}$, C. Timmermans ${ }^{80,79}$, O. Tkachenko ${ }^{40}$, P. Tobiska ${ }^{31}$, C.J. Todero Peixoto ${ }^{18}$, B. Tomé ${ }^{71}$, Z. Torrès ${ }^{35}$, A. Travaini ${ }^{9}$, P. Travnicek ${ }^{31}$, C. Trimarelli ${ }^{56,45}$, M. Tueros ${ }^{4}$, R. Ulrich ${ }^{40}$, M. Unger ${ }^{40}$, L. Vaclavek ${ }^{32}$, M. Vacula ${ }^{32}$, J.F. Valdés Galicia ${ }^{67}$, L. Valore ${ }^{59,49}$, E. Varela ${ }^{63}$, A. Vásquez-Ramírez ${ }^{29}$, D. Veberič ${ }^{40}$, C. Ventura ${ }^{26}$, I.D. Vergara Quispe ${ }^{4}$, V. Verzi ${ }^{50}$, J. Vicha ${ }^{31}$, J. Vink ${ }^{82}$, S. Vorobiov ${ }^{75}$, H. Wahlberg ${ }^{4}$, C. Watanabe ${ }^{25}$, A.A. Watson ${ }^{c}$, M. Weber ${ }^{39}$, A. Weindl ${ }^{40}$, L. Wiencke ${ }^{85}$, H. Wilczyński ${ }^{69}$, M. Wirtz ${ }^{41}$, D. Wittkowski ${ }^{37}$, B. Wundheiler ${ }^{8}$, A. Yushkov $^{31}$, O. Zapparrata ${ }^{13}$, E. Zas ${ }^{78}$, D. Zavrtanik ${ }^{75,76}$, M. Zavrtanik ${ }^{76,75}$, and L. Zehrer ${ }^{75}$

${ }^{1}$ Centro Atómico Bariloche and Instituto Balseiro (CNEA-UNCuyo-CONICET), San Carlos de Bariloche, Argentina

${ }^{2}$ Centro de Investigaciones en Láseres y Aplicaciones, CITEDEF and CONICET, Villa Martelli, Argentina

${ }^{3}$ Departamento de Física and Departamento de Ciencias de la Atmósfera y los Océanos, FCEyN, Universidad de Buenos Aires and CONICET, Buenos Aires, Argentina

${ }^{4}$ IFLP, Universidad Nacional de La Plata and CONICET, La Plata, Argentina

${ }^{5}$ Instituto de Astronomía y Física del Espacio (IAFE, CONICET-UBA), Buenos Aires, Argentina

${ }^{6}$ Instituto de Física de Rosario (IFIR) - CONICET/U.N.R. and Facultad de Ciencias Bioquímicas y Farmacéuticas U.N.R., Rosario, Argentina

${ }^{7}$ Instituto de Tecnologías en Detección y Astropartículas (CNEA, CONICET, UNSAM), and Universidad Tecnológica Nacional - Facultad Regional Mendoza (CONICET/CNEA), Mendoza, Argentina

${ }^{8}$ Instituto de Tecnologías en Detección y Astropartículas (CNEA, CONICET, UNSAM), Buenos Aires, Argentina

${ }^{9}$ Observatorio Pierre Auger, Malargüe, Argentina

10 Observatorio Pierre Auger and Comisión Nacional de Energía Atómica, Malargüe, Argentina

${ }^{11}$ Universidad Tecnológica Nacional - Facultad Regional Buenos Aires, Buenos Aires, Argentina

${ }^{12}$ University of Adelaide, Adelaide, S.A., Australia

${ }^{13}$ Université Libre de Bruxelles (ULB), Brussels, Belgium

14 Vrije Universiteit Brussels, Brussels, Belgium

${ }^{15}$ Centro Brasileiro de Pesquisas Fisicas, Rio de Janeiro, RJ, Brazil

${ }^{16}$ Centro Federal de Educação Tecnológica Celso Suckow da Fonseca, Nova Friburgo, Brazil

${ }^{17}$ Instituto Federal de Educação, Ciência e Tecnologia do Rio de Janeiro (IFRJ), Brazil

18 Universidade de São Paulo, Escola de Engenharia de Lorena, Lorena, SP, Brazil

${ }^{19}$ Universidade de São Paulo, Instituto de Física de São Carlos, São Carlos, SP, Brazil

${ }^{20}$ Universidade de São Paulo, Instituto de Física, São Paulo, SP, Brazil

${ }^{21}$ Universidade Estadual de Campinas, IFGW, Campinas, SP, Brazil

${ }^{22}$ Universidade Estadual de Feira de Santana, Feira de Santana, Brazil

${ }^{23}$ Universidade Federal do ABC, Santo André, SP, Brazil

${ }^{24}$ Universidade Federal do Paraná, Setor Palotina, Palotina, Brazil

${ }^{25}$ Universidade Federal do Rio de Janeiro, Instituto de Física, Rio de Janeiro, RJ, Brazil

${ }^{26}$ Universidade Federal do Rio de Janeiro (UFRJ), Observatório do Valongo, Rio de Janeiro, RJ, Brazil

${ }^{27}$ Universidade Federal Fluminense, EEIMVR, Volta Redonda, RJ, Brazil

${ }^{28}$ Universidad de Medellín, Medellín, Colombia

${ }^{29}$ Universidad Industrial de Santander, Bucaramanga, Colombia

${ }^{30}$ Charles University, Faculty of Mathematics and Physics, Institute of Particle and Nuclear Physics, Prague, Czech Republic

${ }^{31}$ Institute of Physics of the Czech Academy of Sciences, Prague, Czech Republic

32 Palacky University, RCPTM, Olomouc, Czech Republic

${ }^{33}$ CNRS/IN2P3, IJCLab, Université Paris-Saclay, Orsay, France 
${ }^{34}$ Laboratoire de Physique Nucléaire et de Hautes Energies (LPNHE), Sorbonne Université, Université de Paris, CNRSIN2P3, Paris, France

35 Univ. Grenoble Alpes, CNRS, Grenoble Institute of Engineering Univ. Grenoble Alpes, LPSC-IN2P3, 38000 Grenoble, France

${ }^{36}$ Université Paris-Saclay, CNRS/IN2P3, IJCLab, Orsay, France

${ }^{37}$ Bergische Universität Wuppertal, Department of Physics, Wuppertal, Germany

${ }^{38}$ Karlsruhe Institute of Technology (KIT), Institute for Experimental Particle Physics, Karlsruhe, Germany

${ }^{39}$ Karlsruhe Institute of Technology (KIT), Institut für Prozessdatenverarbeitung und Elektronik, Karlsruhe, Germany

${ }^{40}$ Karlsruhe Institute of Technology (KIT), Institute for Astroparticle Physics, Karlsruhe, Germany

${ }^{41}$ RWTH Aachen University, III. Physikalisches Institut A, Aachen, Germany

42 Universität Hamburg, II. Institut für Theoretische Physik, Hamburg, Germany

${ }^{43}$ Universität Siegen, Department Physik - Experimentelle Teilchenphysik, Siegen, Germany

${ }^{44}$ Gran Sasso Science Institute, L’Aquila, Italy

45 INFN Laboratori Nazionali del Gran Sasso, Assergi (L'Aquila), Italy

${ }^{46}$ INFN, Sezione di Catania, Catania, Italy

${ }^{47}$ INFN, Sezione di Lecce, Lecce, Italy

${ }^{48}$ INFN, Sezione di Milano, Milano, Italy

${ }^{49}$ INFN, Sezione di Napoli, Napoli, Italy

${ }^{50}$ INFN, Sezione di Roma "Tor Vergata", Roma, Italy

${ }^{51}$ INFN, Sezione di Torino, Torino, Italy

52 Istituto di Astrofisica Spaziale e Fisica Cosmica di Palermo (INAF), Palermo, Italy

53 Osservatorio Astrofisico di Torino (INAF), Torino, Italy

54 Politecnico di Milano, Dipartimento di Scienze e Tecnologie Aerospaziali , Milano, Italy

55 Università del Salento, Dipartimento di Matematica e Fisica “E. De Giorgi”, Lecce, Italy

${ }^{56}$ Università dell'Aquila, Dipartimento di Scienze Fisiche e Chimiche, L'Aquila, Italy

${ }^{57}$ Università di Catania, Dipartimento di Fisica e Astronomia, Catania, Italy

58 Università di Milano, Dipartimento di Fisica, Milano, Italy

${ }^{59}$ Università di Napoli "Federico II", Dipartimento di Fisica "Ettore Pancini”, Napoli, Italy

${ }^{60}$ Università di Palermo, Dipartimento di Fisica e Chimica "E. Segrè", Palermo, Italy

${ }^{61}$ Università di Roma "Tor Vergata", Dipartimento di Fisica, Roma, Italy

62 Università Torino, Dipartimento di Fisica, Torino, Italy

${ }^{63}$ Benemérita Universidad Autónoma de Puebla, Puebla, México

${ }^{64}$ Unidad Profesional Interdisciplinaria en Ingeniería y Tecnologías Avanzadas del Instituto Politécnico Nacional (UPIITA-IPN), México, D.F., México

65 Universidad Autónoma de Chiapas, Tuxtla Gutiérrez, Chiapas, México

66 Universidad Michoacana de San Nicolás de Hidalgo, Morelia, Michoacán, México

${ }^{67}$ Universidad Nacional Autónoma de México, México, D.F., México

${ }^{68}$ Universidad Nacional de San Agustin de Arequipa, Facultad de Ciencias Naturales y Formales, Arequipa, Peru

${ }^{69}$ Institute of Nuclear Physics PAN, Krakow, Poland

${ }^{70}$ University of Łódź, Faculty of High-Energy Astrophysics,Lódź, Poland

${ }^{71}$ Laboratório de Instrumentação e Física Experimental de Partículas - LIP and Instituto Superior Técnico - IST, Universidade de Lisboa - UL, Lisboa, Portugal

72 "Horia Hulubei” National Institute for Physics and Nuclear Engineering, Bucharest-Magurele, Romania

73 Institute of Space Science, Bucharest-Magurele, Romania

74 University Politehnica of Bucharest, Bucharest, Romania

75 Center for Astrophysics and Cosmology (CAC), University of Nova Gorica, Nova Gorica, Slovenia

${ }^{76}$ Experimental Particle Physics Department, J. Stefan Institute, Ljubljana, Slovenia

${ }^{77}$ Universidad de Granada and C.A.F.P.E., Granada, Spain

${ }^{78}$ Instituto Galego de Física de Altas Enerxías (IGFAE), Universidade de Santiago de Compostela, Santiago de Compostela, Spain

${ }^{79}$ IMAPP, Radboud University Nijmegen, Nijmegen, The Netherlands

${ }^{80}$ Nationaal Instituut voor Kernfysica en Hoge Energie Fysica (NIKHEF), Science Park, Amsterdam, The Netherlands 
${ }^{81}$ Stichting Astronomisch Onderzoek in Nederland (ASTRON), Dwingeloo, The Netherlands

${ }^{82}$ Universiteit van Amsterdam, Faculty of Science, Amsterdam, The Netherlands

${ }^{83}$ University of Groningen, Kapteyn Astronomical Institute, Groningen, The Netherlands

${ }^{84}$ Case Western Reserve University, Cleveland, OH, USA

${ }^{85}$ Colorado School of Mines, Golden, CO, USA

${ }^{86}$ Department of Physics and Astronomy, Lehman College, City University of New York, Bronx, NY, USA

${ }^{87}$ Louisiana State University, Baton Rouge, LA, USA

${ }^{88}$ Michigan Technological University, Houghton, MI, USA

${ }^{89}$ New York University, New York, NY, USA

${ }^{90}$ Pennsylvania State University, University Park, PA, USA

${ }^{91}$ University of Chicago, Enrico Fermi Institute, Chicago, IL, USA

92 University of Delaware, Department of Physics and Astronomy, Bartol Research Institute, Newark, DE, USA

93 University of Wisconsin-Madison, Department of Physics and WIPAC, Madison, WI, USA

${ }^{a}$ Fermi National Accelerator Laboratory, Fermilab, Batavia, IL, USA

${ }^{b}$ Max-Planck-Institut für Radioastronomie, Bonn, Germany

${ }^{c}$ School of Physics and Astronomy, University of Leeds, Leeds, United Kingdom

${ }^{d}$ Colorado State University, Fort Collins, CO, USA

$e^{e}$ now at Hakubi Center for Advanced Research and Graduate School of Science, Kyoto University, Kyoto, Japan

$f$ also at University of Bucharest, Physics Department, Bucharest, Romania 